\title{
«Escuchar a los que saben»: asociaciones agrarias y mediatización de saberes para una nueva agricultura durante la emergencia del agronegocio ${ }^{1}$
}

\author{
Gabriel Fernando Carini²
}

\section{Resumen}

El objetivo general del presente artículo es describir los procesos de recepción y reproducción de los imperativos asociados al agronegocio por parte de las asociaciones agrarias de tipo tradicional, es decir, aquellas abocadas a la defensa y reivindicación de intereses agrarios. En ese marco, proponemos el análisis tanto de la discursividad generada por la dirigencia agraria como de las estrategias diagramadas para mejorar el desempeño económico de sus bases sociales. Partimos de la idea de que estas estrategias de profesionalización del oficio de productor agropecuario fueron un intento por mejorar la performance empresaria de los productores agropecuarios, procurando que incorporen innovaciones en las formas de producir como en las de gestionar sus explotaciones agropecuarias. Este proceso tensionó las funciones históricamente asumidas por las asociaciones gremiales a la par que contribuyó a legitimar diversos tópicos inherentes al nuevo modelo productivo.

Palabras clave: agronegocio - estrategias de profesionalización - asociaciones agrarias discursividad

\begin{abstract}
The general objective of this article is to describe the processes of reception and reproduction of the imperatives associated with agribusiness by traditional agricultural associations, i.e. those dedicated to the defence and vindication of agrarian interests. In this framework, we propose the analysis of both the discursiveness generated by the agrarian leadership and the strategies diagrammed to improve the economic performance of their social bases. We started from the idea that these strategies of professionalization of the profession of agricultural producer were an attempt to improve the business performance of agricultural producers, trying to incorporate innovations in the ways of producing and managing their farms. This process strained the functions historically assumed by the guild associations at the same time that it contributed to legitimize diverse topics inherent to the new productive model.
\end{abstract}

Keywords: agribusiness - professionalization strategies - agrarian associations - discursivity

\footnotetext{
1 Trabajo recibido el 27/10/2018. Aceptado el 19/12/2018.

${ }^{2}$ Universidad Nacional de Río Cuarto. Universidad Nacional de Córdoba. Consejo Nacional de Investigaciones Científicas y Técnicas. Contacto: gcarini@hum.unrc.edu.ar
} 


\section{A modo de introducción}

El agronegocio como forma de producción dominante dentro del heterogéneo mundo rural argentino no constituye una novedad. Aunque algunas de las prácticas agronómicas y de los discursos legitimadores que implicó hunden sus raíces en el momento de la modernización agrícola de la década de 1960, sus rasgos más característicos se terminaron de prefigurar durante la década de 1990. La implementación del paquete tecnológico,sumado a una coyuntura de precios internacionales favorables, propició el vuelco casi masivo hacia la producción de soja. Este proceso trajo aparejado una serie de mutaciones en el paisaje productivo y social del agro pampeano (aunque no se circunscribió exclusivamente a esta región). Así, se aceleró el proceso de liquidación de explotaciones agropecuarias. Para dimensionar esta situación de una manera más gráfica, podemos decir que solo ocho empresas desplazaron de la agricultura a 153 establecimientos de tamaño medio, 72 de la zona núcleo y 81 del resto de la región pampeana (Peretti, 1998).Otro de los fenómenos más visibles fue el crecimiento exponencial de la superficie implantada con soja y el desplazamiento de cultivos y actividades que históricamente habían predominado en los espacios productivos. En el ámbito regional la superficie implantada con la mencionada oleaginosa creció en un $82 \%$. Esto supuso una disminución correlativa de las forrajeras, que pasaron a ocupar la mitad de la superficie implantada que poseían en 1988. Además, hubo importantes cambios en la actividad pecuaria, manifiestos no solo en la pérdida del stock ganadero sino también en las innovaciones en las formas de organizar el trabajo dentro de la explotación agropecuaria y en las innovaciones productivas que se implementaron (Carini, 2014).

A esa dinámica productiva se le adicionó un contexto social y político que favoreció soluciones de tipo neoliberal (Beltrán, 2011).El discurso asociado a la eficiencia en los resultados, a la competitividad de las unidades productivas y a la inserción en los mercados globales se trasladó casi de forma automática para interpretar la realidad del sector agropecuario. Emergió, de esa forma,una discursividad de tipo tecnologizante (Balsa, 2007) que legitimaba las transformaciones que se operaban en la fisonomía de la estructura social agraria. Esta discursividad interpelaba a los productores para que asumieran una actitud empresarial en la organización y gestión de sus unidades productivas y, fundamentalmente, que incorporaran los avances que se registraban en el campo de la biotecnología y la ingeniería genética. En definitiva, construía nuevos 
sentidos sobre las prácticas agropecuarias que se trasladaban en imperativos ineludibles para aquellos productores que quisieran abrirse camino en el contexto productivo (Carini, 2017). Las asociaciones agrarias no permanecieron al margen de estas transformaciones, por el contrario, se constituyeron en espacios estratégicos para la promoción y adopción de las prácticas agronómicas del nuevo modelo productivo. Cobraron centralidad, de esa forma, asociaciones de productores que privilegiaban ya no las instancias de confrontación/negociación con el Estado sino las de formación y divulgación de saberes y prácticas vinculadas a lo que consideraban un nuevo paradigma productivo. La flexibilidad y apertura fueron aspectos constitutivos de los modelos organizativos de la nueva institucionalidad. El primer elemento hace referencia a la posibilidad de incorporar cambios de forma rápida a su estructura organizativa, lo que supuso introducir alternaciones en sus roles y funciones y, en particular, en las modalidades de vinculación con otros actores del agro.El segundo elemento, el carácter abierto de sus esquemas organizacionales, se refiere, por ejemplo, a la incorporación de empresas vinculadas al sector agropecuario como asociadas a dichas instituciones. Si bien la comisión directiva debía (y debe) estar compuesta por productores agropecuarios, que son los que tienen voz y voto en las asambleas, estaba prevista la posibilidad de incorporar como socios a empresas tanto del sector privado como público. Fue justamente esta apertura lo que le posibilitó entablar una excelente relación con las empresas proveedoras de insumos, logrando, de esa forma, ventajas para sus asociados con convenios especiales para el financiamiento en la compra de insumos, a los que se añadieron convenios especiales de financiamiento bancario (Carini, 2018).Lo anterior nos permite inferir cierto distanciamiento existente tanto en el modelo organizativo de las entidades de nuevo cuño como en los roles y funciones respecto de las asociaciones reivindicativas.

En ese marco, la presencia de estas asociaciones «innovadoras» no solo atomizó aún más la estructura de representación de intereses agrarios sino que supuso la instalación de una serie de desafíos que las entidades devenidas en «tradicionales» debieron asumir y resolver. En este sentido, nos interesa historizarcómo estas últimas receptaron y procesaron los imperativos del agronegocio. ${ }^{3}$ Teniendo en cuenta esto, en nues-

${ }^{3}$ Esta situación propició visiones alternativas sobre las dinámicas que se generaron entre estos actores. Algunos entendieron que se produjo una erosión de las bases sociales de las segundas en detrimento de las primeras, lo que las relegó a una situación de marginalidad y 
tra propuesta de investigación cobra relevancia el estudio de lo que denominamos como estrategias de profesionalización, es decir, aquellas iniciativas desplegadas por la dirigencia de las asociaciones gremiales tendientes a mejorar la performance empresaria de sus bases sociales a los fines de garantizar tanto su permanencia y reproducción dentro del proceso productivo como la persistencia de la propia entidad. Dentro de las mismas, a modo de ejemplo, podemos mencionar: la oferta de servicios agronómicos, informáticos y administrativos por parte de las asociaciones agrarias, la generación de negocios agroindustriales con actores nacionales e internacionales, la realización de conferencias, seminarios y talleres a cargo de especialistas, la re-organización del modelo organizativo para adecuarlo a la prestación de servicios vinculados al nuevo modelo productivo, etc.Particularmente, nos centrarnos en aquellas estrategias institucionales que tuvieron como principal objetivo la divulgación mediante soportes materiales de los saberes agronómicos y que, simultáneamente, supusieron la legitimación del nuevo patrón socio-productivo en ciernes.

\section{Algunas precisiones conceptuales y metodológicas}

Nuestra propuesta de investigación se inscribe en el campo de los estudios sociales agrarios, caracterizado por ser un espacio de convergencia entre múltiples disciplinas. A pesar de esto, cobra centralidad una perspectiva de abordaje histórico conducente a establecer continuidades y rupturas en las lógicas institucionales de las asociaciones agrarias «tradicionales»y, más específicamente, en las representaciones que la dirigencia construyó sobre el agronegocio que marcan ciertos desplazamientos tanto en los perfiles socio-económicos de sus bases sociales como en su quehacer institucional. No nos interesa el análisis comunicacional del soporte material en sí mismo -aunque consideraremos algunos elementos sobre el mismo- sino la construcción de representaciones que sobre el agronegocio realizaron las asociaciones agrarias de tipo gremial. En virtud de esto, ocupa un lugar central el análisis de las representaciones e imágenes construidas por las entidades agrarias de corte tradicional. Es

competencia que en ocasiones no pudieron resolver favorablemente (Lattuada, 2006); en cambio, para otros se dio una especie de división del trabajo que no alteró la composición de las entidades tradicionales (Gras, 2009). 
decir, nuestra investigación se concentra en un plano estrictamente discursivo. Esto no implica desconocer que queda afuera de nuestro estudio un significativo conjunto de prácticas institucionales que complementan y completan el análisis de los fenómenos discursivos pero implica asumir que los mismos poseen cierta materialidad que se traduce en la posibilidad de incorporar pautas conductuales en los individuos.

Sobre esa base, seleccionamos como objeto de análisis las prácticas discursivas de una entidad gremial de primer grado, la Sociedad Rural de Río Cuarto (en adelante SRRC). En la elección de este referente empírico secontemplaron diferentes cuestiones de orden teórico y metodológico. En primer lugar, por su perfil institucional -es decir, todos aquellos aspectos ligados a su estructura interna y externa- la SRRC responde al tipo ideal de entidad gremial. Desde su creación a principios del siglo XX, y con mayor vigor desde su consolidación a mediados de la década de 1930, se encontraba abocada estrictamente a la defensa de los intereses de los productores ganaderos asociados a ella y poseía una frágil estructura de servicios que se restringía a la provisión de guías ganaderas. Además, se encontraba (y encuentra) adherida a la Confederación de Asociaciones Rurales de la Tercera Zona (CARTEZ) y, por su intermedio, a las Confederaciones Rurales Argentinas (CRA). Todas instancias supralocales de articulación de intereses agrarios.

En segundo lugar, nos interesa enfocarnos en las capas de productores previamente «incluidas», los que ya habían entrado en el proceso de capitalización y tecnificación (Murmis, 1998, p. 234). Estos sujetos sociales son los que conforman la base social de la SRRC. ${ }^{4}$ Este sector no solo se compone de lo que en la sociología rural se conoce como agricultura familiar ${ }^{5}$ sino que abarca a un conjunto de sujetos sociales que igualmente constituyen un factor decisivo en el mantenimiento de formas de vida rural modernizada pero con presencia local. Estos sujetos han recibido diferentes denominaciones que enfatizan en laconjunción entre diversos aspectos, en especial en lo relativo a la incorporación de tecnologías, a las dimensiones y modalidad de gestión de sus explota-

\footnotetext{
${ }^{4}$ Para ampliar la trayectoria histórica de la entidad, las características socio-económicas de sus bases sociales, sus demandas y discursos se puede consultar: Carini (2011, 2014 y 2015). ${ }^{5}$ No existe una definición unívoca de agricultura familiar y los alcances históricos y actuales de dicha categoría constituyen un tema ampliamente debatido en el campo de los estudios rurales. Generalmente, el productor familiar se define por relaciones sociales en las que las variables básicas son el control sobre la tierra (en propiedad o usufructo) y el uso de la mano de obra familiar (Murmis, 1998).
} 
ciones y a los vínculos de sus explotaciones con diferentes territorios: productores tradicionales capitalizados (Barsky y Gelman, 2009, p. 494), productores tradicionales reconvertidos (Flood, 2005, p. 146), o productores territorializados (Gras y Hernández, 2013, p. 53). Estos productores conservan bajo su propiedad los espacios productivos y mantienen el control sobre el proceso productivo, lo que no excluye la contratación de maquinarias ni la ampliación de superficies productivas mediante el arriendo. Puede tratarse de empresas de mediana o gran escala, con cierto grado de diversificación territorial, por lo que poseen vínculos con distintos territorios, es decir, que dependen de diversas dinámicas locales. Fue, precisamente, en las franjas medias y superiores de la estructura agraria que algunos investigadores han observado un corrimiento hacia formas más empresariales en los perfiles socio-económicos que históricamente caracterizaron a los productores comprendidos en ese sector (Gras, 2009; Gras, 2010; Hernández, 2009; Gras y Hernández, 2009; Gras y Hernández, 2013; Muzlera, 2009).

Finalmente, en tercer lugar, consideramos que emprender el estudio propuesto apelando a la reducción de la escala permite un abordaje más exhaustivo de las transformaciones que imprimió la vigencia del agronegocio en las lógicas institucionales de las entidades gremiales. En este sentido, cabe advertir que este trabajo no pretende ser representativo o constituirse en una muestra de casos más generales sino que intenta abordar singularidades para demostrar cómo esas especificidades pueden en todo caso contradecir y de-construir los casos globales, más que representarlos fielmente a la manera positivista clásica. No obstante, esto no implica abandonar la pretensión de generalidad a partir de los estudios micro, puesto que los mismos pueden contribuir a revelar aspectos fundamentales sobre los fenómenos generales.

En relación al corpus de nuestro análisis,nos concentramos en el estudio de la columna institucional de la SRRC, Información Ruralista, publicada los días viernes en el Suplemento Agropecuario Tranquera Abierta del diario Puntal. La colección de este diario puede consultarse en la Hemeroteca del Archivo Histórico Municipal de Río Cuarto. Se compone de un total de 266 columnas publicadas entre el 29 de abril de 1994 y el 28 de diciembre de 2001. Cabe aclarar que durante el mes de enero no se editaba el Suplemento Agropecuario y que en algunos casos hubo ediciones especiales (que se corresponden generalmente a la primera quincena del mes de septiembre, después de realizada la feria anual de invierno de la SRRC). El período seleccionado resulta significativo, 
pues durante el mismo se operó una serie de procesos que evidencian la vigencia de un nuevo modelo socio-productivo. Así, se asistió a una reasignación de recursos de la ganadería a la agricultura, evidenciada en la re-estructuración productiva de ambas actividades, aunque más contundente en la segunda. En este sentido, se incorporaron nuevas tecnologías y modalidades de producir que no solo alteraron las formas históricas en las que se venía realizando sino que supusieron un significativo incremento de escala para las unidades productivas. La arista más visible fue la liberación comercial de la soja genéticamente modificada y la adopción del paquete tecnológico. Paralelamente, se eliminó una tupida red de organismos y mecanismos que le permitían al Estado regular los mercados productivos y se asistió a un marcado debilitamiento de organismos que, como el Instituto Nacional de Tecnología Agropecuaria (INTA), habían cumplido un rol vital en la innovación productiva. Más allá de esto, lo que nos interesa remarcar es que, en conjunto, estos procesos señalaron para las asociaciones agrarias tradicionales una serie de desafíos que debieron afrontar y resolver para garantizar su permanencia y la de sus bases sociales.

La sistematización y el procesamiento del corpus fueron realizados sobre la base de los siguientes pasos y criterios. En primer lugar, realizamos un relevamiento del total de las columnas aparecidas durante la delimitación temporal que hemos propuesto, consignando la fecha de aparición, la página del suplemento agropecuario en donde se encuentra y el título y subtítulo en los casos en que se encontraba expresado. Sobre esa base realizamos una matriz de registro de la información (Perona, 2000) donde ordenamos los datos según un criterio cronológico. En segundo lugar, consideramos importante realizar una primera lectura de IR y consignar a qué género periodístico correspondía tomando en cuenta tres posibilidades según la propuesta de Peralta y Urtasun (2004):1) informativos, aquellos que buscan transmitir datos, hechos o sucesos (crónica, nota informativa, entrevista, investigación, portada, foto documental, notas de color y secciones fijas); 2) los de opinión, cuya función es transmitir y formar opinión sobre los datos, hechos o sucesos (editoriales, columnas de opinión, análisis, panoramas, reseñas y foto editorial); y 3) los de entretenimiento, aquellos que se orientan, precisamente, a entretener o distraer (humor gráfico, verbal, horóscopos, juegos y literatura). Nuestro corpus se compone de 117 (43,98\%) columnas de carácter informativo, $147(55,26 \%)$ de opinión y $2(0,76 \%)$ en las que se combinan elementos de opinión e información por igual que nos im- 
piden clasificarlo en uno u otro rubro. Esta operación es importante para distinguir aquellos términos, vocablos y expresiones que son empleados asiduamente y que nos permitieron identificar nudos, marcas y señales en los discursos (Vasilachis de Gialdino, 1997 y 2007) vertidos a través de la columna institucional de la SRRC.

Una vez construida esa matriz de información, en tercer lugar, avanzamos en clasificar las columnas según la discursividad a la que se las puede inscribir. Para ello, retomamos la caracterización que Balsa (2007) realizó de las formaciones discursivas a partir de los discursos públicos del sector agropecuario entre los años 2004 y $2006 .{ }^{6}$ El autor identifica tres formaciones discursivas en el discurso de los actores agrarios. Una, la liberal-conservadora, caracterizada por poner énfasis en la cuestión del respeto al derecho inalienable a la propiedad de la tierra y por pregonar la libertad total de los mercados. En este discurso no se diferencian sujetos agrarios según el tamaño de sus explotaciones ni sus formas de tenencia del suelo. Otra, agrarista, cuyo núcleo histórico ha sido la crítica a la situación de concentración de la propiedad de la tierra como principal traba para el desarrollo agrario nacional y causa del sufrimiento de los pequeños productores. Este es un discurso que brinda gran importancia a la diferenciación social de los productores, tanto por la tenencia del suelo, como por el tamaño de las explotaciones o el volumen de su capital. Finalmente, una tecnologizante, que se concentra en la celebración del avance tecnológico como el elemento central de las virtudes y problemas del sector. A partir de ello, construimos redes semánticas, es decir, un conjunto de términos, de palabras, de vocablos, de ítems lexicales que se reiteran en un texto y que refieren a actores, relaciones, contextos, procesos, fenómenos, estados, objetos (Vasilachis de Gialdino, 2007). Estos enunciados forman una unidad en la medida en que se refieran a un solo y mismo objeto, por lo que esa unidad está dada por el espacio en el que los diversos objetos se perfilan y continuamente se transforman. El objeto, lejos servir de referencia para vincular un conjunto de enunciados, está constituido, más bien, por el conjunto de esas formulaciones (Foucault, 1968). Bajo esos criterios se puede reconocer

\footnotetext{
${ }^{6}$ Cabe aclarar que creemos válida la posibilidad de trasladar estas formaciones discursivas a temporalidades anteriores porque representan formas históricas por medio de las cuales se han expresado diferentes actores agrarios, especialmente las asociaciones agrarias. En este sentido, el mismo autor ha empleado estas nociones para indagar la discursividad de las asociaciones agraria en otros períodos históricos, especialmente, en la primera mitad del siglo XX (Balsa, 2012 y 2013).
} 
que en el período 1994-2001 los enunciados contenidos en la columna institucional de la SRRC responden en su mayoría a una formación discursiva tecnologizante (109 columnas que representan el 40,97\% del total). ${ }^{7}$ Sobre estas columnas centramos nuestro análisis, puesto que indican un desplazamiento en las formas históricas de interpelar a las bases sociales de la entidad y concebir la actividad agropecuaria.

Otro elemento importante para la interpretación de esta información es la noción de convergencia discursiva enunciada por Vasilachis de Gialdino(2007), la cual se opera cuando se reiteran las representaciones por dos o más actores y son empleados sus expresiones, términos y vocablos en las argumentaciones públicas en un mismo espacio temporal. Utilizamos esta idea en dos sentidos: por un lado, para mostrar que las formaciones discursivas que hemos caracterizado en ciertos contextos históricos pueden actuar de forma complementaria y, por otro lado, para advertir que las mismas no son exclusivamente una cualidad del discurso de las asociaciones agrarias. Pueden encontrarse, por ejemplo, en los posicionamientos de los partidos políticos, en propuestas de técnicos o, incluso, en la prensa. Esta cuestión permite potenciar discursos, ocluir alternativas y, sobre todo, imponer, sostener y legitimar un determinado deber ser sobre la actividad agropecuaria. ${ }^{8}$

De lo anterior se desprende que nuestro trabajo adopta una estrategia metodológica cualitativa, entendiendo como sus rasgos más sobresalientas el interés por el significado y la interpretación, el énfasis sobre la importancia del contexto y de los procesos y la estrategia inductiva y hermenéutica (Vasilachis de Gialdino, 2007). Para ello apelamos a la técnica de análisis de discurso. Si bien ésta puede remitir a un conjunto heterogéneo de procedimientos y de referencias teórico-conceptuales con ella hacemos referencia a la perspectiva que terminó de conformarse como campo durante la década de 1960 en Francia y que pone énfasis en el análisis de la articulación del discurso con sus «condiciones de producción» (Bonetto y Martínez, 1995).

\footnotetext{
${ }^{7} \mathrm{La}$ formación discursiva tecnologizante presente en IR es seguida por la liberal-conservadora con 103 que representan un $38,73 \%$ y se completa con un $0,75 \%$ ( 2 columnas) que constituye una combinación de la formación agrarista con la tecnologizante. El resto, 52 columnas $(19,55 \%)$, no fue posible inscribirlo en ninguna de las formaciones discursivas que utilizamos. Asimismo, dentro de las columnas pertenecientes a la formación discursiva tecnologizante predomina el género de opinión, con 63 columnas $(57,80 \%)$.

${ }^{8}$ En sentido similar Poggi (2011) siguiendo a Wodak (2003) utiliza la idea de intensificación cuando el enunciador del discurso pretende modificar la situación epistémica de su proposición intensificándola en relación a la fuerza ilocucionaria de las afirmaciones.
} 


\section{En torno al oficio de productor: saberes y habilidades para una nue- va agricultura}

Se ha sostenido que la vigencia del agronegocio implicó una serie de desplazamientos en relación con las formas de concebir la actividad agropecuaria que se tradujeron en modalidades de operar dentro del proceso productivo radicalmente distintas respecto de etapas anteriores (Hernández, 2009; Gras y Hernández, 2009 y 2013). Mirados en conjunto, estos procesos acentuaron el carácter empresario de la actividad agropecuaria, la complementación de habilidades y saberes de diferentes ámbitos del conocimiento y la necesaria integración de lo rural con lo industrial. En ese marco, articuladas en la idea de la reconversión empresaria, desde Información Ruralista comenzaban a realizarse referencias a esos desplazamientos y, por ende, a socializar un conjunto de saberes y prácticas vinculados al agronegocio. Un lugar destacado lo ocupaba la necesidad de procurar un manejo empresarial de las explotaciones agropecuarias como plataforma indispensable no solo para alternar exitosamente en los mercados internacionales sino también para asegurar la reproducción de las mismas. ${ }^{9}$ Así, las instancias de profesionalización de la actividad agropecuaria generadas por la SRRC poseían su contrapartida en la faz discursiva que alertaba sobre las mutaciones en el negocio agropecuario y, por ende, en la acción institucional:

(...) por cuanto Argentina está inmersa en un mundo que ha cambiado. Esta Sociedad Rural de Río Cuarto continuará ejerciendo su democrática posición de defensa del sector agropecuario (...) Pero también sabemos que nuestra acción deberá encaminarse al mejoramiento de [la] vida de nuestras empresas rurales. ${ }^{10}$

Esta suerte de 'toma de conciencia' de la dirigencia rural se asentaba discursivamente en el imperativo de garantizar una transformación en las estructuras productivas de las empresas agropecuarias de los productores asociados a la SRRC que ahora debía orientarse a incrementar la productividad a través de la incorporación de tecnología y a realizar un uso «inteligente» de las habilidades administrativas y financieras:

Ya nadie puede estar ajeno a la profunda transformación que viene

\footnotetext{
${ }^{9}$ Información Ruralista, 18/11/1994.
}

${ }^{10}$ Información Ruralista, 24/07/1998. 
dándose en los sistemas agroalimentarios. Esta deviene, indudablemente, como consecuencia de la creciente globalización de las economías mundiales, modificando las estructuras productivas, industriales y fundamentalmente comerciales de todos los países, que ya no admiten posicionamientos que pretendan asimilarse o no involucrarse en estos cambios. (...) Las empresas rurales se ven obligadas a producir cada días más, para lo que deben adecuar sus estructuras, actualizándolas y preparándolas para esa mayor producción. Ello significa también la necesidad de acceder a la moderna tecnología e implica necesariamente una mayor capitalización. Pero ésta debe ser racional, ordenada y oportuna, de acuerdo con la propia envergadura de cada empresa. No siempre esa tecnología implica grandes inversiones, pero sí alcanzar una estructura adecuada, que permita los cambios que deben darse en el andamiaje de la empresa, preparándola para acometer el desafío de nuestros tiempos. ${ }^{11}$

Finalizaba la editorial afirmando que se estaba en presencia de una «verdadera revolución [que] se da no solamente en los sistemas productivos. Esto se continúa, tiene una natural correlación, en el desenvolvimiento empresario». Esta noción implicaba, para la dirigencia rural de la SRRC, hacer un uso adecuado de las opciones que ofrecían los mercados y recurrir a «una buena y oportuna asesoría». ${ }^{12}$ Sin embargo, lo anterior no nos debe llevar a pensar que los elementos del discurso tecnologizante fueron apropiados de forma plena por la dirigencia rural de la entidad. Por un lado, se insistía en prestar atención a los «nuevos requisitos» de la actividad agropecuaria, mejorar eficiencia y productividad a partir de los avances tecnológicos. Por el otro lado, existían en la columna institucional referencias constantes a ser cautelosos con endeudarse más allá de los límites que permitía la propia empresa. Esta situación se ponía de manifiesto especialmente con la cuestión de la tecnología, que se consideraba como un «espejismo»:

iCuánta cosa buena que hay para mejorar nuestra producción agropecuaria! Empero, debemos ser cautelosos a la hora de tomar decisiones, Los productores ya lo saben, pero siempre es oportuno formular alguna prevención o reflexión: mucho de lo que apreciamos en las parcelas agrícolas demostrativas, no deja de ser un espejismo. Son cultivos o pasturas logradas así con recursos que no se tienen en

${ }^{11}$ Información Ruralista, 24/07/1998.

${ }^{12}$ Información Ruralista, 24/07/1998. 
el campo: riego, encanalado del suelo, fertilizadas al máximo, con cuidados a lo largo de su evolución, que bien merecen el calificativo de 'bebés malcriados'. Con 'todos los chiches', por adoptar una expresión popular. Esa tecnología, adaptada a nuestras reales posibilidades, podrá ser asimilada paulatinamente, como sucede con todos los adelantos, pero no debemos pretender tener lujos inmediatamente. iNo nos dejemos encandilar! En cuanto a la maquinaria agrícola, también hay que contemplar su eventual adecuación al dimensionamiento de nuestros campos y a nuestras necesidades iy posibilidades!. ${ }^{13}$

Cobraba relevancia en ese contexto la apelación a estrechar vínculos transectoriales que atendieran a una diversificación de la red de producción en virtud de las cualidades-especificidades de consumidores ubicados en los más diversos mercados internacionales. Recordemos que uno de los pilares del nuevo modelo agropecuario lo constituyó la posibilidad de trasvasar las fronteras de lo rural e integrar distintos actores y componentes de la cadena productiva que agregaran una mayor complejidad al negocio agrícola (Hernández, 2009; Gras y Hernández, 2013). Para la dirigencia de la SRRC, esto se traducía en un llamado para que sus asociados incrementaran su «empresarialidad», procurando el desarollo de emprendimientos agroindutriales:

(...) debemos pensar en emprendimientos que permitan procesar la materia prima que se obtiene en nuestros campos. Favorecer la radicación de agro-industrias sobre la base de los productos alimenticios que tenemos a nuestro alcance. Río Cuarto, insistimos, es un importante $-\mathrm{y}$ natural- centro dinamizador de la economía regional, con base agropecuaria. Es un núcleo comercial proveedor de insumos para el campo de una dilatada región. Pero, ha llegado la hora de pensar en la transformación de nuestra producción primaria, en el procesamiento de esos bienes en la industrialización. Más precisamente en la agro-industria. Procesar, en el lugar en el que se da la producción, incorporándole mano de obra e inversiones de capital, procurando un mayor movimiento económico general. He aquí, entonces, el gran desafío de la hora actual. ${ }^{14}$

${ }^{13}$ Información Ruralista, 21/03/1997. Ver también las columnas de los días 17/05/1995 y 11/10/1996.

${ }^{14}$ Información Ruralista, 08/03/1996. 
Otro núcleo de la discursividad generada desde la columna institucional de la SRRC fue el que se abocó a comentar las competencias y saberes que debían considerarse para enfrentar la actividad productiva como un negocio. En este sentido, se generó un discurso con una alta performatividad empresaria, es decir, una enunciación que legitimaba prácticas que consideraba adecuadas en el nuevo contexto productivo y que pretendía generar un cambio en las modalidades de operar en el proceso productivo de las bases sociales de la entidad. En ese discurso la eficiencia constituía la coordenada sobre la cual se asentaba la garantía de la reproducción de la empresa agropecuaria y, a su vez, planteaba una fuerte convergencia discursiva -en términos de Vasilachis (2007)-con la discursividad de entidades como AACREA y AAPRESID. ${ }^{15}$ Esto implicaba no solo atender a mejorar las técnicas y demás aspectos atinentes a lo productivo sino también perfeccionar lo relativo a la comercialización de la producción. En ese proceso, resultaba esencial el asesoramiento especializado, tal como podemos apreciar en el siguiente fragmento:

Si nos sentimos eficientes, debemos procurar serlo más, mejorando nuestros sistemas de producción, delineando estrategias superadoras de la actual situación, con vistas a mejorar rendimientos que, a su vez, nos permitan acceder a una mejor posición vendedora de lo que producimos. Debemos estar alertas e informados sobre el desenvolvimiento, las perspectivas y la evolución de los mercados. Deberemos practicar mejores técnicas de producción. Ver lo que hace el vecino y cómo lo hace, si es que éste obtiene mejores resultados que nosotros. Escuchar a los técnicos que demuestran reales conocimientos y una sabia experiencia, con resultados a la vista. Tener un panorama global. Estamos inmersos en un mundo cada vez más competitivo y cada vez más exigente. No podemos aislarnos. Por el contrario, debemos actuar en consecuencia. ${ }^{16}$

Así, desde el discurso de la dirigencia ruralista se comenzaba a construir la necesidad de incorporar al conocimiento como un nuevo factor (decisivo) para llevar adelante el proceso productivo. Esta situación era advertida casi de forma constante en las páginas de Información Ruralista, que al respecto comentaba:

\footnotetext{
15 Ver: Gras y Hernández (2013a) y Liaudat (2015).

${ }^{16}$ Información Ruralista, 18/11/1994.
} 
(...) los productores dedican sus esfuerzos para la adecuada y oportuna implantación de cultivos de cosecha gruesa (...). Estas tareas suponen inversiones, fuertes erogaciones sobre las que necesariamente deben tomarse decisiones empresarias racionales, suficientemente analizadas y evaluadas, dado que sobre este basamento económico-financiero, deben sustentarse todos los planes de producción del campo. Hoy en día, deben tenerse en cuenta todas las estimaciones que cada ejercicio requiere. En el caso de la agricultura, se deben tomar las lógicas y razonadas evaluaciones, sobre la base de datos actualizados de mercado y sus perspectivas, para lo que será indispensable una correcta información y una confiable asesoría. Así, al momento de adquirir semillas y agroquímicos, (...) deberemos analizar y evaluar precios, condiciones, calidad de la mercadería, financiamiento, etc. (...) Se deberá disponer de una información actualizada sobre líneas de créditos, con sus respectivos plazos, intereses, montos prestables, etc. Será también necesaria la actualización técnica sobre rendimientos que ofrecen las distintas variedades, tareas culturales, ciclos de cada semilla, su aptitud productiva, su tolerancia frente a plagas, enfermedades, malezas, etc. En cuanto a los agroquímicos, herbicidas, plaguicidas, fertilizantes, etc. su debido conocimiento técnico sobre su correcta aplicación, etc. (...) creemos que la actualización, la información, el análisis y la evaluación económicos, son presupuestos que nadie debe desdeñar. ${ }^{17}$

De forma más enfática se sostenía: «Debemos hacernos cada día más empresarios. Acudir a la información actualizada, asesorarnos convenientemente, conocer la situación de los mercados». ${ }^{18}$ Se argumentaba de esta forma sobre la necesidad de reconvertir la actividad agropecuaria en una de tipo empresarial y asumir roles más activos en relación con el conocimiento. El carácter empresario era asociado a la toma de decisiones basadas en el manejo de información sobre tópicos que excedían lo meramente productivo. De esta manera, se consideraba fundamental para el perfil socio-productivo del productor agropecuario tanto la atención de los diversos componentes del campo financiero como la inevitabilidad de recurrir al asesoramiento de técnicos y especialistas en diversas áreas del conocimiento.

Para la dirigencia de la SRRC la actividad agropecuaria ya no consistía en el dominio de habilidades meramente agronómicas sino que

${ }^{17}$ Información Ruralista, 10/10/1997.

${ }^{18}$ Información Ruralista, 10/05/1996. 
incluía una vasta gama de competencias. Se trataba de una actividad cada vez más profesionalizada donde el conocimiento sobre la nueva coyuntura productiva y financiera requería de una actualización permanente con el fin de asegurar la performance empresaria de los productores. En un extenso fragmento que transcribimos a continuación, la dirigencia de la SRRC daba cuenta de esta ruptura y abogaba por la necesidad de abandonar el «conservadorismo»y asumir un «manejo empresario»:

Quizá por tradición, por conservadorismo o por su propia naturaleza, el productor ha sabido manejarse siempre con capacidad e inteligencia en los diversos sistemas productivos, pero -ahora más que nunca- muestra serias falencias en lo que hace a su manejo empresario ‘Cómo se advierte esto? En un posicionamiento no del todo firme frente a los mercados, en donde debe comercializar su producción (...) Los mercados ya no son los mismos de algunos años atrás. Se han diversificado. Actúan de manera distinta. En nuestros días se manejan múltiples opciones para la venta de productos primarios o procesados, industrializados. En materia de granaria, debemos conocer opciones, mercados de futuro, etc. en cuanto a la ganadería, los otrora grandes mercados concentradores van perdiendo su hegemonía y en cambio existe la posibilidad de vender en campo, en ferias locales, etc. Debemos conocer todas estas variables y operar con las que nos ofrezcan mejores posibilidades. Pero no solo debemos conocer todo sobre comercialización. Debemos igualmente saber manejar los créditos, es decir los diversos elementos de financiación de la empresa rural. En definitiva: escuchar a los que saben. Asesorarse. ${ }^{19}$

Existía en la dirigencia una percepción sobre la necesidad de amoldar las prácticas de las bases sociales de la entidad a la nueva realidad productiva. En su discurso se encontraba presente la convicción de un quiebre respecto a la forma de concebir la actividad agropecuaria. Así, a diferencia de un pasado no tan lejano, el éxito de la empresa rural ya no dependía de la habilidad de su titular para «manejarse en los distintos sistemas productivos». Este tipo de conocimiento heredado de generación en generación, como lo plantea Hernández (2009), se tornó superfluo. El imperativo del nuevo contexto productivo requería de la decisiva intervención de los saberes de técnicos y especialistas. La dirigencia de la

${ }^{19}$ Información Ruralista, 19/04/1996. 
SRRC entendía que ya no bastaba con el saber agronómico sino que apelaba a que éste fuese complementado con diferentes capacidades.

\section{A modo de conclusión}

La configuración del agronegocio señaló más rupturas que continuidades en el mundo rural argentino. Dentro de estas últimas se pueden mencionar aquellas ligadas a la acentuación del proceso de agriculturización iniciado en la década de 1960, la incorporación de avances generados por el campo de la ingeniería genética y la biotecnología y el aumento de las escalas necesarias para transitar con éxito por el proceso productivo. No obstante, durante el último cuarto del siglo XX las rupturas se proyectaron con mayor intensidad, cuestión que llevó tanto a investigadores como a técnicos del sector a plantear la vigencia de un nuevo paradigma. Más allá de los matices que se le pueden realizar a esta afirmación, existieron indicios que habilitaron pensar en una nueva instancia dentro del capitalismo agrario en Argentina. Si bien -como dijimos- la agriculturización y la incorporación de tecnología no constituyeron una novedad en el paisaje rural nacional, las dimensiones que estos factores adquirieron sí lo fueron. El crecimiento exponencial de las oleaginosas en general y del cultivo de la soja en particular alteró radicalmente las prácticas agronómicas y, por ende, las dinámicas territoriales de los sujetos agrarios. Esta situación no fue exclusiva de la agricultura sino que aconteció en otras actividades como en la ganadería, donde también se registraron transformaciones en los manejos de los rodeos que plantearon modalidades de operar por fuera y por dentro de las «tranqueras» sustancialmente diferentes a etapas anteriores.

Así, la incorporación de avances e innovaciones como la siembra directa o las semillas transgénicas junto a otras de tipo organizacionales hicieron -cada vez más- de la actividad agropecuaria una de tipo conocimiento-intensivo. En este sentido, se colocó el acento en la necesidad de incorporar saberes de diferentes áreas al «negocio» agrícola. Esto no solo se plasmó en la introducción de nuevas tecnologías sino también en la mediación (decisiva) de profesionales y técnicos que sumaron su experticia al desenvolvimiento económico de las unidades productivas, lo que generó nuevas solidaridades entre actores que no necesariamente estaban familiarizados o sus trayectorias sociales vinculadas al mundo rural. Bajo ese imperativo el nuevo modelo de desarrollo agrario supuso 
un desplazamiento del saber heredado en favor de un conjunto de habilidades que trasvasaron lo agrario. Es decir, el «oficio» de productor agropecuario asumió nuevos contornos que estuvieron ligados a una racionalidad empresaria, lo que habilitó a pensar a quienes internalizaron esos imperativos como «empresarios innovadores»: flexibles, capaces de alterar sin dificultad la planificación productiva de las empresas agropecuarias, atentos a los requerimientos globales de los mercados, volcados a la introducción de nuevas capacidades y saberes que adicionaran complejidad al negocio agrícola, en definitiva, capaces de orbitar por fuera de los límites de lo local.

Con ese trasfondo, la profesionalización de la actividad agropecuaria iniciada por la SRRC demandó la necesidad de validar la adopción de nuevos saberes y habilidades. Esa operación fue realizada mediante la estructuración de una estrategia discursiva que tuvo una alta performatividad empresaria, puesto que procuró estimular el cambio en las pautas y comportamientos productivos de las bases sociales de la entidad, apelando a que asumieran las labores productivas como unas de tipo empresarial. Se propiciaba, en definitiva, un quiebre en la concepción de la actividad agropecuaria. La construcción de este nuevo imaginario se mediatizó, fundamentalmente, a través de la prensa gráfica. Este soporte no solo posibilitó comunicar las actividades institucionales a las bases sociales sino que además le permitía a la dirigencia ruralista alcanzar a un público más amplio. Así, Información Ruralista, la columna institucional de la SRRC, se mostró como un mecanismo difusor eficaz para cimentar la profesionalización del oficio de productor agropecuario.

En esta operación se encontraba implícita una modalidad distinta de interpelación de las bases sociales que incorporaba componentes discursivos diferentes a los que históricamente habían caracterizado la enunciación de la entidad. La dirigencia de la SRRC acudió a enunciados, frases y conceptos de corte tecnologizante. Eficiencia, tecnología, cálculo racional, manejo empresario, asesoramiento, fueron las coordenadas desde las cuales se procuró producir un cambio en las formas tradicionales de concebir (y operar) dentro y fuera del proceso productivo. En un plano discursivo, para la dirigencia de la SRRC se trataba, fundamentalmente, de un cambio en las estructuras comerciales y productivas que volvían superflua la experticia meramente agronómica. Bajo esa idea rectora, por un lado se hacía hincapié en que los productores prestasen atención a las perspectivas y modalidades de negocios que proponían los merca- 
dos nacionales e internacionales. Esto era una pre-condición de la actividad productiva -tan importante como la fertilización de los suelos- que aseguraba la comercialización y, por ende, la rentabilidad de lo producido. Por otro lado, era preciso tener presente que la actividad agropecuaria constituía una cuestión de especialistas. Hubo una apelación constante destinada a remarcar el carácter excluyente que poseía el asesoramiento técnico. Éste no se limitaba solo a lo agronómico sino que abarcaba otras competencias como lo legal, lo contable, lo administrativo, que necesariamente debían resolverse «tranqueras afuera». También en esa forma de concebir la actividad agropecuaria residía la convicción de que se debía tener en cuenta no solo lo que ocurría «tranqueras adentro» sino que cada vez ocupaba más importancia lo que acontecía por afuera, con la comercialización de la producción. Estas argumentaciones -de carácter constante- vertidas en la columna institucional constituían la contracara de un conjunto de conferencias, seminarios, demostraciones, capacitaciones, etc. que habían re-significado las funciones asumidas por la SRRC. De esta manera, el discurso operaba como un medio que reforzaba la idea del cambio que se estaba llevando a cabo en el modelo organizativo de la entidad y que también era indispensable reproducir en las explotaciones agropecuarias de los asociados a la entidad.

En definitiva, las respuestas de la SRRC frente a un contexto que implicaba profundas transformaciones en la dinámica productiva supuso la necesidad de ofrecer una respuesta activa por parte de la dirigencia agraria para garantizar la propia reproducción institucional. Así, los cambios en los discursos y las prácticas fueron verso y anverso de una misma estrategia institucional por medio de la cual no solo se vehiculizó una determinada construcción simbólica sobre lo rural sino que, concomitantemente, se erigió además a la columna Información Ruralista como un canal por el que penetraron y se legitimaron los saberes ligados al nuevo paradigma productivo.

\section{Referencias bibliográficas}

Balsa, J. (2007). Las disputas hegemónicas en torno de las cuestiones sociales agrarias de la pampa argentina en la actualidad. En N. Girbal-Blacha y S. Mendonça(Coords.). Cuestiones agrarias en Argentina y Brasil, pp. 149-170. Buenos Aires, Argentina: Prometeo. 
Balsa, J. (2012). Formaciones discursivas y disputas por la hegemonía en torno a los modelos de desarrollo agrario. En S. Lázzaro y J. Balsa (Coords.). Agro y politica en Argentina. El modelo agrario en cuestión, 1930-1943, pp. 35-117. Buenos Aires, Argentina: CICCUS.

Balsa, J. (2013). Los avatares de la reforma de la ley de colonización durante el primer peronismo (1946-1955). En N. Girbal-Blacha y S. Mendonça (Dirs.). Corporaciones agrarias y politicas públicas en América Latina, pp. 193-226. Rosario, Argentina: Prohistoria.

Flood, C. (2005). Trayectorias institucionales comparadas de innovación tecnológica en la región pampeana. En R. Benencia y C. Flood (Comps.). Trayectorias y contextos. Organizaciones rurales en la Argentina de los noventa, pp. 137-177. Buenos Aires, Argentina: La Colmena.

Beltrán, G. (2011). Las paradojas de la acción empresaria. Las asociaciones del empresariado argentino y la persistencia de las reformas estructurales. En A. Pucciarelli (Coord.). Los años de Menem. La construcción del orden neoliberal, pp. 221-262. Buenos Aires, Argentina: Siglo XXI.

Carini, G. (2011). Intereses agrarios y mediación corporativa en el sur de Córdoba. El discurso de la dirigencia agraria ante la nueva política peronista. En E. Escudero y R. Camaño (Comps.). Rio Cuarto en tiempos del primer peronismo. Aproximaciones desde la Historia, pp. 191-228. Córdoba, Argentina: Ferreyra.

Carini, G. (2014). Reivindicaciones, discursos y estrategias en mutación: la Sociedad Rural de Río Cuarto en el marco de una 'nueva agricultura' (1990-2000). En G. Olivera (Dir.). El agro cordobés en el siglo XX: entramados productivos, politicos y sociales desde una perspectiva histórica, pp. 223-280. Córdoba, Argentina: Editorial de la Secretaria de Investigación, Ciencia y Técnica de la Facultad de Filosofía y Humanidades de la Universidad Nacional de Córdoba.

Carini, G. (2015). Nuevo Estado, viejos intereses: corporaciones agrarias y mediación política en el interior de Córdoba (1935-1955). Estudios del ISHIR, 5 (11), 93-113. Recuperado de: http:// web2.rosario-conicet.gov.ar/ojs/index.php/revistaISHIR/article/ view/421

Carini, G. (2017). «Cada día más empresarios»: asociaciones de pro- 
ductores, agronegocio y estrategias institucionales en la pampa cordobesa (1995-2002). Sociobistórica. Cuadernos del CISH, (40), 120 .

Recuperado de: https://www.sociohistorica.fahce.unlp.edu.ar/article/ view/SHe032

Carini, G. (2018). Agro, negocio y nueva institucionalidad en las pampas: itinerarios y propuestas de abordajes para el análisis de la representación de intereses agrarios. En G. Banzato,G. Blanco y J. Perren(Comps.). Expansión de la frontera productiva y estructura agraria argentina, siglos XIX-XX, pp. 417-439. Buenos Aires, Argentina: Prometeo.

Flood, C. (2005). Trayectorias institucionales comparadas de innovación tecnológica en la región pampeana. En R. Benenciay C. Flood (Comps.). Trayectorias y contextos. Organizaciones murales en la Argentina de los noventa, pp. 137-177. Buenos Aires, Argentina: La Colmena.

Gras, C. (2009). El nuevo empresariado agrario: sobre la construcción y los dilemas de sus organizaciones. En C. Gras y V. Hernández (Coords.). La Argentina rural. De la agricultura familiar a los agronegocios, pp. 215-237. Buenos Aires, Argentina: Biblos.

Gras, C. y Hernández, V. (2009). El fenómeno sojero en perspectiva: dimensiones productivas, sociales y simbólicas de la globalización agrorrural en la Argentina. En C. Gras y V. Hernández. (Coords.). La Argentina rural. De la agricultura familiar a los agronegocios, pp. 15-39. Buenos Aires, Argentina: Biblos.

Gras, C. y Hernández, V. (2013). Los pilares del modelo agribusinessy sus estilos empresariales. En C. Gras y V. Hernández (Coords.). El agro como negocio. Producción, sociedad y territorios en la globalización, pp. 17-49. Buenos Aires, Argentina: Biblios.

Gras, C. y Hernández, V. (2013a). Asociatividad del empresariado agrícola en Argentina. AACREA y AAPRESID en perspectiva. En J. Muzleray A. Salomón (Coords.). Sujetos sociales del agro argentino. Configuraciones históricas y procesos de cambio, pp. 36-67. Rosario, Argentina: Prohistoria.

Hernández, V. (2009). La ruralidad globalizada y el paradigma de los agronegocios. En C. Gras y V. Hernández (Coords.). La Argentina rural. De la agricultura familiar a los agronegocios, pp. 39-65. Buenos Aires, Argentina: Biblos. 
Lattuada, M. (2006). Acción colectiva y corporaciones agrarias en la Argentina. Transformaciones institucionales a fines del siglo XX. Bernal, Argentina: Universidad Nacional de Quilmes.

Liaudat, M. (2015). La construcción hegemónica de las entidades técnicas en el agro argentino: análisis de los discursos de AAPRESID y AACREA en la última década. Mundo Agrario, 16 (32), 1-32. Recuperado de: http://www.mundoagrario.unlp.edu.ar/article/view/ MAvl6n32a04

Murmis, M. (1998). Agro argentino: algunos problemas para su análisis. En N. Giarracca y S. Cloquell (Comps.). Las agriculturas del Mercosur. El papel de los actores sociales, pp. 205-211. Buenos Aires, Argentina: La Colmena.

Muzlera, J. (2009). Chacareros del siglo XXI. Herencia, familia y trabajo en la pampa gringa. Buenos Aires, Argentina: Imago Mundi.

Peralta, D. y Urtasun, M. (2004). La crónica periodistica. Buenos Aires, Argentina: La Crujía.

Peretti, M. (1998). Competitividad de la empresa agropecuaria argentina en la década de los '90. En Actas de las XXIX Reunión Anual de la Asociación Argentina de Economía Agraria, pp. 1-20. Buenos Aires, Argentina: AAEA-Universidad Nacional de La Plata.

Perona, N. (2000). La construcción de un código. Supuestos conceptuales en el registro de observables. Cinta de Moebio, (13), 126-132. Recuperado de http://www.revistas.uchile.cl/index.php/CDM/article/viewFile/26191/27483

Poggi, M. (2011). Problemática agraria y prensa escrita en la argentina de los años 70. Representaciones y debates sobre la propiedad de la tierra. Tesis doctoral en Ciencias Sociales y Humanas de la Universidad Nacional de Quilmes.

Saltalamacchia, H. (1997). Los datos y su creación. Caguas, Puerto Rico: Kryteria.

Vasilachis de Gialdino, I. (1997). La construcción de representaciones sociales: el discurso politico y la prensa escrita. Barcelona, España: Gedisa.

Vasilachis de Gialdino, I. (2007). Condiciones de trabajo y representaciones sociales. El discurso político, el discurso judicial y la prensa escrita a la luz del análisis sociológico-lingüístico del discurso. Discurso y Sociedad, I (1), 148-187. Recuperado de: http://www.dissoc. org/ediciones/v0ln01/DS1(1)Vasilachis.pdf 